\title{
EL CONCEPTO DE NATURALEZA EN LOS ESTUDIANTES DE LA UNALM
}

\author{
THE CONCEPT OF NATURE IN STUDENTS UNALM
}

\author{
${ }^{1}$ Armando E. Díaz Barba
}

\begin{abstract}
Resumen
Uno de los conceptos de mayor reiteración en la UNALM es el de naturaleza. Desde los cursos hasta las investigaciones, pasando por las diferentes formas de proyección social, estudiantes y profesores estamos permanentemente enfrentados en el debate abierto o tácito sobre este concepto. El artículo busca conocer cuál es la definición o las definiciones que yendo más allá de las preferencias fomentadas por el sentido común es o son las que ha o van internalizado tanto los estudiantes como los profesores. Sobre esa base se busca saber si sus estudios los hizo cambiar o reafirmar su concepto de naturaleza y si creen que la UNALM debería manejar una única definición de naturaleza.
\end{abstract}

Palabras Clave: Naturaleza, sociedad, universidad, estudiantes.

\begin{abstract}
Nature is one of the most reiteration concepts in UNALM. From courses to research and going through various forms of social work, students and professors are constantly faced within the open or tacit debate about this concept. This article tries to know what is the definition or definitions, which go beyond preferences fostered by common sense, and if they are being internalized by both students and professors. On this basis we want to know if their studies change or reaffirm their concept of nature, and if they think UNALM should handle a unique definition of nature.
\end{abstract}

Key words: Nature, Society, University, Students

\section{Introducción.}

Un tema que ha resurgido con mucha fuerza a raíz de diversos eventos de origen antropogénico que acarrean cambios ambientales es el de la relación de la sociedad (el hombre) con el ambiente (naturaleza). El paradigma dicotómico de dos entidades diferentes que es un axioma del mundo occidental cuya aceptación generalizada lo exoneraba de mayores discusiones no solo en las ciencias naturales sino también en ciencias sociales aparentemente parece haber colapsado por lo que desde diferentes disciplinas se está replanteando la urgencia de traer al debate la forma cómo se entienden esas relaciones en otras culturas para lo cual es imprescindible identificar y precisar el contenido que dan a cada uno de los términos. Dentro de ellos el que parece presentar mayores complicaciones de entendimiento es el concepto naturaleza por lo que amerita discutir su contenido entre otros por tres motivos puntuales: 1 . Desde lo académico, tomar nota de los presupuestos epistemológicos de las concepciones de naturaleza, 2. Desde el campo de la ética, contrastar las noción de naturaleza de la cultura occidental con las de otras culturas a fin de evaluar la conveniencia de introducir en la primera algunos elementos que podrían conducir a un cambio del paradigma eminentemente consumista por otro donde el fin sea el hombre y no el consumo y 3. Un punto de vista más práctico remite a la urgencia de llegar a la comprensión, dar el tratamiento pertinente y lograr soluciones adecuadas a los crecientes conflictos sociales por problemas ambientales especialmente los que se dan entre las grandes empresas y las poblaciones locales toda vez que se ha convertido en un potencial campo de trabajo para los/as egresados/as de diferentes especialidades de la UNALM para el cual deben estar altamente preparados.

Siendo el objetivo del presente trabajo conocer cuál es el concepto de naturaleza para los estudiantes de la UNALM independiente de la especialidad y de los ciclos que cursan, el artículo hace un breve recuento de los contenidos de ese término a través de la historia para luego presentar lo que los estudiantes entienden por naturaleza.

\section{Materiales y métodos}

HIPÓTESIS: En el estudiante de la UNALM predomina el concepto de naturaleza como aquello que no ha sido intervenido por el hombre, siendo este el principal y único atributo para ser considerado como tal. De esta manera se atiene solamente al paradigma occidental como resultado de un eslabonamiento conceptual que se arrastra desde épocas preuniversitaria adquiridas ya sea en la escuela, en el colegio o en el hogar y otros ámbitos de socialización del alumno.

La segunda hipótesis afirma que la universidad no ha generado modificaciones sustantivas en los estudiantes respecto al concepto de naturaleza. 


\section{METODOLOGIA}

La población en estudio está constituida por los 5130 estudiantes de la UNALM matriculados el ciclo 2013-2.

El estudio se desarrolló empleando dos técnicas:

1. Focus Gruop, Se trabajó con 4 grupos, cada grupo estaba integrado por 6 estudiantes.

Tiempo de duración de las sesiones: Entre 60 y 80 minutos.

El moderador fue el responsable de la investigación.

El lugar de las sesiones a pedido de los propios estudiantes fueron los jardines de la universidad Selección de participantes: Grupos heterogéneos: ciclos, géneros, carreras, origen de los ancestros.

2. Un muestreo probabilístico utilizando el diseño del Muestreo Estratificado por facultades.

En este muestreo se ha considerado un margen de error del 5\% y un nivel de confianza del 95\%. Para determinar la dispersión de la variable más trascendente, es decir el concepto de naturaleza, se utilizó un muestreo piloto que comprendió a 130 alumnos elegidos aleatoriamente entre los alumnos de todas las facultades, resultando que la proporción de la cual depende dicha variabilidad igual a 0.813

El tamaño de muestra fue obtenido de acuerdo a la siguiente expresión:

$$
\begin{gathered}
n_{0}=\frac{Z_{(1-\alpha / 2)}^{2} \pi(1-\pi)}{E^{2}} \\
n=\frac{n_{0}}{1+\frac{n_{0}}{N}}
\end{gathered}
$$

Donde:

$Z_{(1-\alpha / 2)}$ : Es el cuantíl $(1-\alpha / 2)$ de la distribución Normal Estándar y que para la investigación se utilizó nivel de confianza de $(1-\alpha) * 100=95 \%$, es decir $Z_{(0.975)}=1.96$.

П: Es la proporción de estudiantes que indica una respuesta adecuada acerca del concepto de naturaleza, obtenida en una muestra piloto de 130 estudiantes y que resultó igual a 0.813

E:Representa el margen de error que en el estudio fue igual al 5\% $(\mathrm{E}=0.05)$

$\mathrm{n}$ :Es el tamaño de muestra definitivo, $\mathbf{n}=\mathbf{2 2 4}$

$\mathrm{n}_{0}$ :Es el tamaño de muestra preliminar, sin corregir por el tamaño de la población, $\mathrm{n}_{0}=234$

$\mathrm{N}$ : Es el tamaño de la población, $\mathrm{N}=5130$

Obtenido el tamaño total de la muestra, se determinó el de tamaño de muestra para cada facultad, utilizando una asignación proporcional al tamaño del estrato.
Tabla 1 Población por facultad y muestra.

\begin{tabular}{lcc}
\hline \multicolumn{1}{c}{ Facultad } & Población (ni) & Muestra (ni) \\
\hline Agronomía & 975 & 43 \\
Ciencias & 805 & 35 \\
Ciencias Forestale & 444 & 19 \\
Economía y Plan. & 957 & 42 \\
Industrias Alimen. & 526 & 23 \\
Ingeniería Agrícol & 523 & 23 \\
Pesquería & 436 & 19 \\
Zootecnia & 464 & 20 \\
\hline \multicolumn{1}{c}{ Total } & 5130 & 224 \\
\hline
\end{tabular}

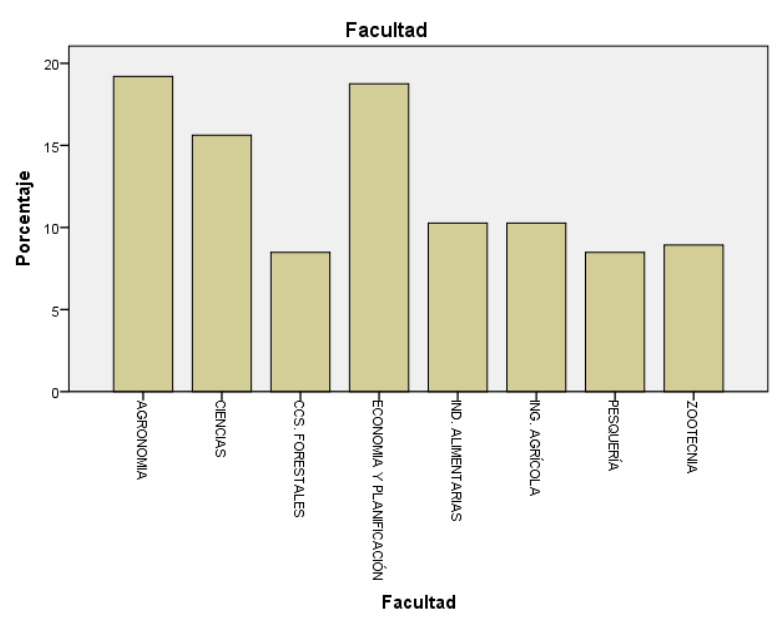

Figura 1. Se tomó la precaución de incluir a estudiantes de todas las especialidades o carreras que se dan en la UNALM.

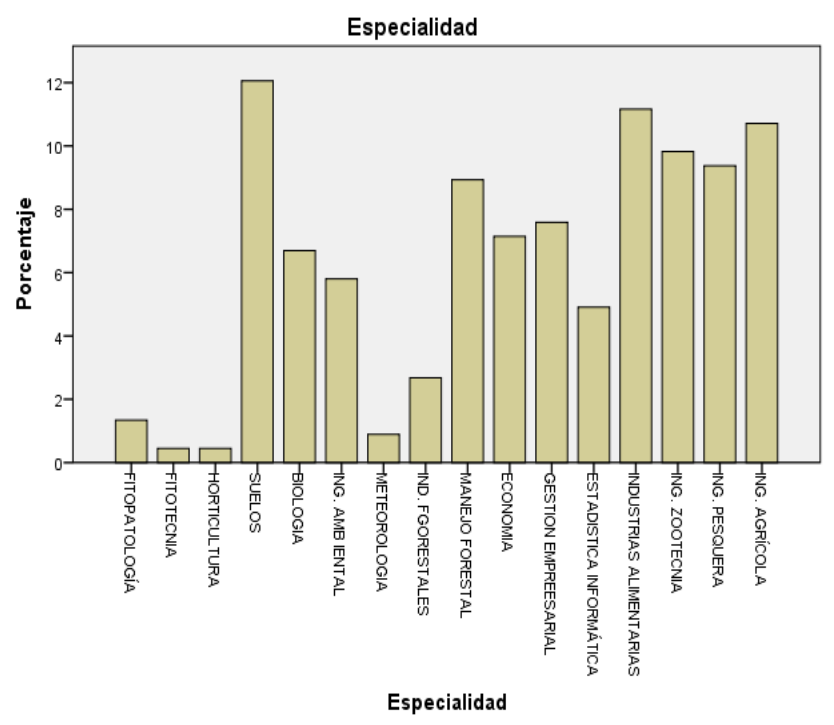

Figura 2. Estudiantes encuestados por especialidad. 
Tabla 2. Estudiantes encuestados por especialidad.

\begin{tabular}{lcc}
\hline \multicolumn{1}{c}{ Especialidad } & Frecuencia & \% Válido \\
\hline Fitopatología & 3 & 1.3 \\
Fitotecnia & 1 &, 4 \\
Horticultura & 1 &, 4 \\
Suelos & 27 & 12,1 \\
Biología & 15 & 6,7 \\
Ing. Ambiental & 13 & 5,8 \\
Meteorología & 2 &, 9 \\
Ind. Forestales & 6 & 2,7 \\
Manejo Forest. & 20 & 8,9 \\
Economía & 16 & 7,1 \\
Gest. Empre. & 17 & 7,6 \\
Estad. Inform. & 11 & 4,9 \\
Inds. Aliment. & 25 & 11,2 \\
Ing. Zootec. & 22 & 9,8 \\
Ing. Pesquera & 21 & 9,4 \\
Ing. Agrícola & 24 & 10,7 \\
\hline \multicolumn{1}{c}{ Total } & 224 & 100,0 \\
\hline
\end{tabular}

El instrumento de recopilación de información ha sido la encuesta, esta contenía preguntas cerradas y abiertas.

Tomado en cuenta los criterios del tiempo de estudios en la universidad y participación en prácticas de campo, se ha dividido la muestra en tres estratos:

1. Inicial, del primer al tercer ciclo.

2. Medio, del cuarto al sexto ciclo.

3. Superior, del séptimo al decimo ciclo.

El tiempo de estudios en la universidad permite identificar: a). Al grupo de estudios generales, que tienen cursos de biología y de ciencias sociales donde el tema naturaleza es recurrente por lo que se asume que el debate por tal concepto debe ser permanente.

b). Al grupo que ya inicio sus cursos de carrera en los que se asume que el componente naturaleza está presente en la mayoría de ellos.

c). Al grupo que esta egresando o próximos a egresar y que se supone que tienen una idea ya formada del concepto naturaleza.

El criterio participación en prácticas de campo, que por ser de carácter obligatorio en muchos cursos de carrera permite a los estudiantes no solo interactuar con sectores sociales de culturas diferentes sino a valorar ya sea favorable o desfavorablemente muchas de sus manifestaciones culturales.

Tabla 3. Ciclos que cursan los estudiantes encuestados.

\begin{tabular}{rcc}
\hline \multicolumn{1}{c}{ Ciclo } & Frecuencia & \%Válido \\
\hline Del 1 al 3 & 10 & 4.5 \\
Del 4 al 6 & 117 & 52.2 \\
Del 7 al 10 & 97 & 43.3 \\
\hline \multicolumn{1}{c}{ Total } & 224 & 100.0 \\
\hline
\end{tabular}

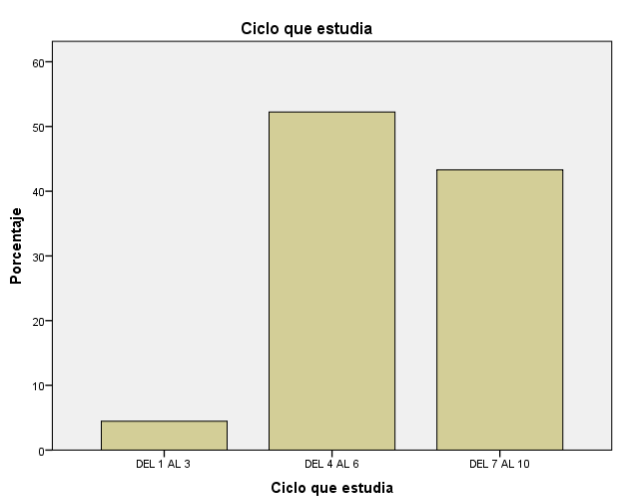

Figura 3. El estudio tomó en cuenta a estudiantes de ambos géneros, siendo mayor el porcentaje femenino.

Tabla 4. Porcentaje por genero de estudiantes encuestados

\begin{tabular}{lcc}
\hline \multicolumn{1}{c}{ Género } & Frecuencia & \% Válido \\
\hline Femenino & 121 & 54.0 \\
Masculino & 103 & 46.0 \\
\hline
\end{tabular}

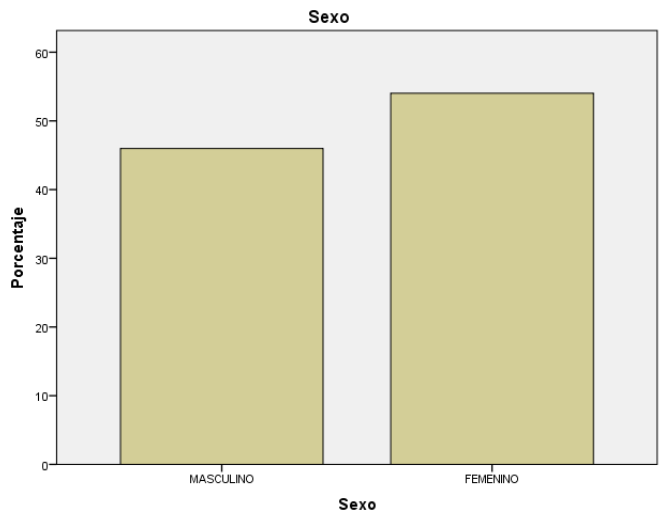

Figura 4. Porcentaje por género de estudiantes encuestados.

\section{Los contenidos del concepto naturaleza a través del tiempo}

Se sabe que desde los albores de la humanidad el hombre ha vivido pendiente de la naturaleza, así (Heyd: 2011: 19) señala: "desde tiempos inmemoriales los seres humanos han tenido en consideración las fuerzas naturales que pueden afectar sus destinos", asociando esta relación "al mundo de las creencias y pautas sociales" (Cerpa: 1996:134), que muchas veces pasarán a formar parte del corpus de mitos que se encargarán de sancionar un tipo relaciones hombre/ambiente ya sea "identificando nuestro origen a partir de especies, en otros creando antagonismos o reglamentando el uso de algunas especies" (Morán: 1990:19). Las reflexiones y teorías sobre las interacciones del mundo social con el mundo natural tienen su partida de nacimiento en "los tiempos clásicos" (Kwiatkowska;2006: 154), como resultado del contacto 
entre la civilización griega y otras culturas (Morán;1990: 29). Simplificando la diversidad de enfoques se pueden encontrar dos tipos de relaciones: uno de separacióndomino sustentado en la total diferenciación de lo humano con la naturaleza que adjudica al hombre el deber, la necesidad y la obligación de controlar y dominar al ambiente natural para pertrecharse de productos que garanticen su subsistencia y otro de unidad donde naturaleza y sociedad son una misma realidad en la que ninguna de ellas prevalece o ejerce dominio sobre la otra. En este tipo de relación ambas dimensiones se necesitan por lo que el eje de la relación es el respeto entre ellos. Para este enfoque la subsistencia humana se da en un marco de armonía entre el hombre y la naturaleza.

\section{Sociedad-naturaleza, una unidad.}

La relación sociedad-naturaleza como entes diferenciados ha atravesado tres etapas según Cerpa (Ibid:135): 1. el espacio concreto, 2. el espacio mítico y 3. el espacio geométrico, es en el primero de ellos, que corresponde a la sociedad primitiva (paleolítico y mesolítico) donde el hombre tiene un visión netamente práctica, por eso se llama espacio de acción, donde "el hombre asigna al territorio (naturaleza) características y funciones semejantes a las suyas propias". En el medioevo el pensamiento dominante sostenía la existencia de un mundo, "superior al terrenal ... en el cual recién llegaremos a ser completos" (Trazegnies: 1997:98), Dios ejercía un control total sobre todas las actividades del hombre, éste se sentía avasallado, subordinado a una naturaleza que imponía sus ritmos y creaba temores entre los hombres obligándolos a refugiarse en él en busca de respuestas a sus interrogantes. Era la fuerza de la religiosidad (sagrado) que se había impuesto desde casi los albores de la humanidad ${ }^{2}$. Durante este período no había separación radical entre naturaleza y sociedad; de existir habría sido muy diferente de la que se da en la modernidad (Pálsson: 2001:82), esta autor cita a Gurevich (199:297) para quien en esa época "el hombre se consideraba a sí mismo como parte integrante del cosmos (...) Su relación con la naturaleza era tan inmensa y completa que no podía mirarla desde afuera; estaba dentro de ella". De acuerdo a Heyd, (Ibid:23), "las poblaciones que han cultivado estrechas relaciones con la fuerzas naturales... frecuentemente se perciben a si mismas como parte del medio ambiente natural y

${ }^{2}$ El peso de la religiosidad en la cosmovisión de algunos pueblos mantiene su vigencia como lo constatan Varas y Williams en la zona de Ayaviri, en las alturas del departamento de Lima, "la cosmovisión campesina está formada por tres dimensiones intimadamente relacionadas en constante tensión y en busca de equilibrio. Estás son: la sociedad humana, la naturaleza y los seres sobrenaturales. En un contexto de crisis, de ruptura de equilibrio entre sociedad humana y naturaleza, los comuneros recurren a rituales como un medio para restituir ese equilibrio". La aparición súbita de grandes masas de roedores que asolaron los campos agrícolas del distrito de Ayavirí en al año 1994, según ellas corresponde a esa ruptura de equilibrio y que luego de varias experiencias de erradicación frustradas los campesinos tuvieron que acudir a un ritual religioso para restituir el equilibrio. (Varas y Williams, "Los rituales para superar conflictos en ecosistemas campesinos". Rev. Tierra Nuestra, Año 15, No 8, Departamento de Ciencias Humanas, UNALM, 2010. partícipes de su transformación, de tal manera que no tratan a la naturaleza como mero recurso u obstáculo".

En la Baja Edad Media se experimentan algunos cambios trascendentales. En la dimensión humana surgen ideas desacralizadoras, el hombre va dejando de depender de Dios, se siente hacedor de su vida, se da cuenta que "puede también tener gratificaciones en este mundo, sin que ello sea pecado; es capaz de progresar, es capaz de producir y de gozar los bienes que resulten de su trabajo" (Trazegnies:Ibid:98). Se inicia un proceso de secularización. Surge la ciencia moderna que va desarrollando métodos de investigación más precisos y se introduce el concepto de tecnología para aprovechar mejor la naturaleza. Esta deja de ser "la madre exigente que nos envuelve, nos marca las pautas de nuestra conducta a las cuales debemos simplemente someternos... se muestra más bien como un entorno que debe ser transformado para ponerlo al servicio de los humanos" (Trazegnies:Ibid:101), lo que se logrará solamente si el hombre consigue dominarla. El fin último será hacerla producir ilimitadamente. En este contexto, Moran (Ibid:19) menciona "que la ideología judeocristiana basada en el libro de la Génesis, donde 'Dios dio al hombre el mando sobre la naturaleza', es la base del comportamiento utilitario de las sociedades de esa tradición", afirmación que induce a pensar que otras sociedades como podrían ser las orientales seguidoras de la tradición budista buscan la armonía entre estas dos dimensiones algo que no necesariamente es cierta ya que como lo afirma Reboratti (2000: 151) “en Japón y China se pueden encontrar algunos de los ejemplos más brutales de destrucción ambiental". Para Kwiatkowska (2006: 156) el enfoque mencionado por Morán resulta "del mito, comúnmente aceptado, que cuenta que antes del advenimiento de la era industrial el ambiente se encontraba totalmente prístino e impoluto". En efecto, afanes destructivos de diversos ecosistemas anteceden a la era industrial tal como lo reporta Bordehore (s/f) al mencionar las prácticas agrícolas inadecuadas que ocasionaron graves problemas de erosión de suelos durante el Imperio Romano, la prohibición de sobreexplotar diversas especies de animales y árboles en Mongolia, en el siglo XIII y la prohibición en Inglaterra en 1306, de la quema de carbón con la consecuente contaminación atmosférica. Igualmente, Sempere y Riechmann (2000), refieren que desde la antigüedad el hombre ha ido haciendo no solo modificaciones sino graves daños al entorno biofísico como el acontecido en el Paleolítico Superior, en Mezherich (Ucrania) donde se levantaron tres estructuras aparentemente con fines ceremoniales con 15 toneladas de huesos de mamut cada una de ellas. Citan a Diamond (1994) quien afirma que algunos pueblos preindustriales considerados ejemplos de conservacionísmo "exterminaron especies, destruyeron ecosistemas y socavaron las bases de su propia existencia durante miles de años". En la misma línea Kwiatkowska (2006:157) menciona estudios que dan cuenta del impacto que las culturas Paleolíticas y Mesolíticas tuvieron en el 
ambiente. Es posible que estos impactos negativos que el propio hombre ha generado en el ambiente sea el origen del interés por conocer la relación que este tiene con la naturaleza, al menos para el mundo occidental, así lo entienden Morán (1990:39).

Es en el período del Renacimiento, donde tendrán notable influencia las ideas de René Descartes y Francis Bacon sobre la ciencia, el método científico, la naturaleza, etc. (Gudynas: 2004). Al Organum de Aristóteles, Bacon contrapone el Novum Organum en el que postula a la ciencia como praxis (acción) destacando que su objetivo es el dominio de la naturaleza a la vez que propone al método deductivo como el método científico por excelencia. A decir de (Bof: 1997) ${ }^{3}$ estos postulados baconianos buscan "subyugar a la naturaleza, presionarla para que nos entregue sus secretos, atarla a nuestro servicio y hacerla nuestra esclava". Por su parte, Descartes con su prédica orientaba al hombre a ser jefe, amo poseedor de la naturaleza (Boff: Ibid) ${ }^{4}$. Para Schumacher(1983:14), esta separación se debe a los cambios filosóficos y religiosos en la actitud del hombre occidental respecto a la naturaleza que se dio en los últimos tres o cuatro siglos, originando que el hombre no se sienta "parte de la naturaleza sino más bien como una fuerza externa destinada a dominarla y conquistarla".

La invasión europea a América Latina no solo es de hombres sino también de ideas que por diversos medios tratarán de ser impuestas, muchas lograrán enraizar en estos territorios, otras no tendrán el mismo éxito en tanto que a más de 500 años de la invasión es posible demostrar el vigor de las visiones locales siendo una de ellas el de la naturaleza cuyo significado y contenido precolombino no ha perdido vigencia en muchas culturas americanas. No resulta exótico por lo tanto afirmar que el actual rechazo a las empresas extractivas parece tener un fuerte componente de pervivencia de estas cosmovisiones locales en las que naturaleza y sociedad es una sola dimensión. En gran parte del mundo andino "la naturaleza se presenta como un ser con vida, tanto como un hermano humano runa. En las tradiciones orales andinas animales, vegetales y humanos intercambian formas que los confunden en una totalidad cósmica" (Cáceres; 2005: 258). En las obras de José María Arguedas abundan ejemplos de esta concepción, así en "Los Ríos Profundos", en el capitulo "El Viejo", Ernesto le manifiesta a su padre que las piedras además de hablar, "parece que caminan, que se revuelven" o en la novela "Todas las Sangres", los seres que describe, "integran la naturaleza en un mundo total y armonioso e integran también el mundo afectivo de los seres humanos, el cual a su turno se integra en él", esta visión que "ordena el mundo en una totalidad", corresponde a una dimensión especial de racionalidad: la racionalidad mítica (López Maguiña; 2005: 245),

${ }^{3}$ El autor consigna: Citado según J. Moltmann, Dios en la creación, Sígueme, Salamanca, 1987.

${ }^{4}$ Textualmente "maître et possesseur de la nature". El autor consigna: Discurso del método, sexta parte, Espasa-Calpe, Madrid, 1986, 84 ss.
Diversos autores han reportado la vigencia actual en muchas culturas de los bosques lluviosos amazónicos de cosmovisiones donde no hay separación de lo humano con lo natural (Rengifo: 1995, citado por Gudynas: 2004; Descola: 1998, 2001; Gudynas: 2004; Tiwi Paati: 2006; Rivera: 2009; Gasché y Vela: 2012). Para estos pueblos la naturaleza junto al hombre forma una sola entidad. Vilcapoma (2008) encuentra en la "solidaridad mística" que se da en la actividad de la caza el surgimiento de esta cosmovisión, de "la creencia que el hombre es semejante al animal y viceversa". Así, los jíbaros consideran a la mayoría de las plantas y animales como personas "que viven en sus propias sociedades y se relacionan con los humanos de acuerdo con ciertas reglas de comportamiento social: los animales de cacería son tratados como afines a los hombres, mientras que las plantas cultivadas son tratadas como parientes por las mujeres" (Descola y Pálsson; Ibid: 18). Heyd (2011:23), al referirse a poblaciones que mantienen estrechas relaciones con las fuerzas de la naturaleza señala que "se rigen por sistemas de creencias y valores que se expresan en prácticas materiales y formas de modificar el entorno que supone que la naturaleza y los seres humanos están integrados en una misma comunidad", por lo que estas poblaciones no perciben a la naturaleza como meros recurso u obstáculo, el autor resalta el concepto de "paisajes sensibles" de Cruikshank en tanto que la consecuencia ética de esta noción "consiste en que se deja de tratar la diversidad de componentes del medio ambiente como meros recursos y que el conjunto de éstos, que incluye a los seres con vida y a los elementos inanimados llegan a ser percibidos como homólogos a los seres humanos por su capacidad de actuación autónoma".

Para Chrif (s/f: 4) aceptar que el indígena amazónico se sienta parte de la naturaleza, es equivocado, las "reiteradas infracciones a las normas, consignadas en sus mitos de origen, que regían su vida cuando eran una unidad con el mundo natural", afirman lo contrario, que se reconocen diferentes a la naturaleza a pesar de seguir manteniendo una relación muy intima con ella. Según este autor el accionar de estos pueblos no obedece al concepto de control y dominio de la naturaleza que caracteriza a la sociedad occidental, por el contrario, suelen actuar guiados por el "cumplimiento de la norma de respeto de no tomar más (animales o plantas) que lo necesario para vivir".

\section{Resultados y discusión}

A la pregunta ¿qué entiende por naturaleza?, se puede encontrar dos grupos de respuestas: uno, la mayoría (76.1\%) responde que es aquello que no ha sido creado por el hombre, o donde este no ha tenido intervención alguna. Se puede hablar de consistencia de este porcentaje en tanto que en el focus grup la mayoría de los participantes concibe de una manera similar a la naturaleza. Ambos casos permite constatar la alta presencia de la visión occidental en la formación de los estudiantes. 
Otro grupo importante aunque con un porcentaje mucho menor considera que tanto el hombre como la naturaleza forman un solo ente, queda claro que en esta visión no hay una separación del mundo natural con el mundo social tal como lo entienden hasta ahora muchas sociedades andinas y amazónicas.

Tabla 5. Que entiende por naturaleza.

\begin{tabular}{lcc}
\hline \multicolumn{1}{c}{ Concepto } & Frecuencia & \%Válido \\
\hline $\begin{array}{l}\text { Todo lo no creado por el } \\
\text { hombre }\end{array}$ & 169 & 76,1 \\
$\begin{array}{l}\text { Nosotros y todo lo que nos } \\
\text { rodea }\end{array}$ & 28 & 12,6 \\
$\begin{array}{l}\text { Esencia donde se desarrolla el } \\
\text { hombre }\end{array}$ & 9 & 4,1 \\
Creación de Dios & 4 & 1,8 \\
Lo natural & 4 & 1,8 \\
Un sistema vivo & 2 &, 9 \\
Algo propio & 2 &, 9 \\
$\begin{array}{l}\text { Espacio donde nos } \\
\text { encontramos }\end{array}$ & 1 &, 5 \\
$\begin{array}{l}\text { Interacción hombre-naturaleza } \\
\text { No contesta }\end{array}$ & 1 &, 5 \\
\hline
\end{tabular}

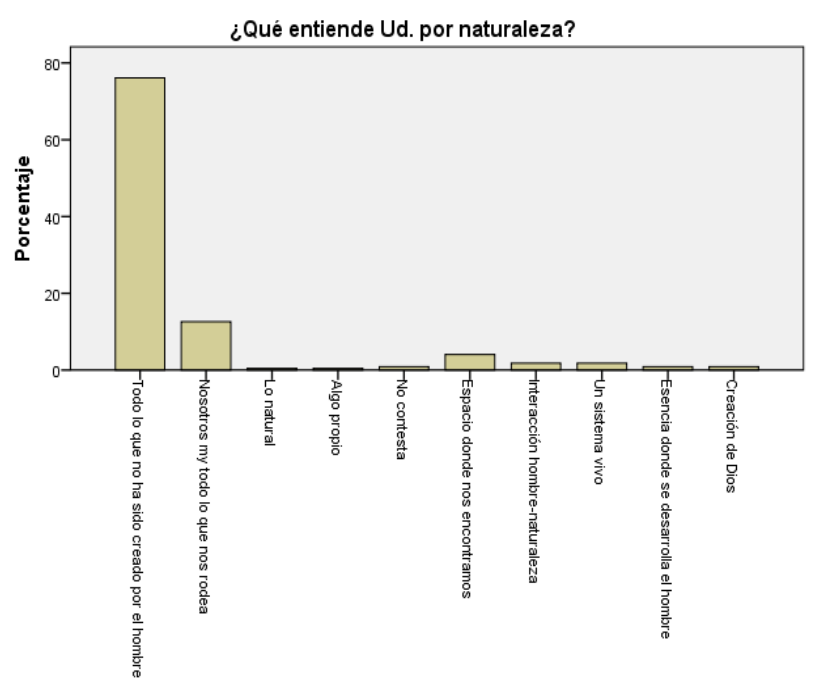

Figura 5. Que entiende por naturaleza.

Se encuentra una gran dispersión de otras respuestas llamando la atención el bajo porcentaje de personas que la consideran el origen divino de la naturaleza, es decir como creación de Dios, posiblemente sea producto del proceso de secularización de los estudiantes molineros.
Tabla 6. Sentido mas frecuente de naturaleza en los cursos.

\begin{tabular}{lcccc}
\hline \multicolumn{1}{c}{ Acepción } & & Frecuencia & \% Válido \\
\hline $\begin{array}{l}\text { Donde el hombre no ha } \\
\text { intervenido }\end{array}$ & & & & 49 \\
$\begin{array}{l}\text { Lo que existe sin separar al } \\
\text { hombre }\end{array}$ & 72 & 33.3 \\
$\begin{array}{l}\text { Interacción hombre-naturaleza } \\
\text { Lo creado por Dios }\end{array}$ & 39 & 18.1 \\
No responde & 6 & 2.8 \\
\hline
\end{tabular}

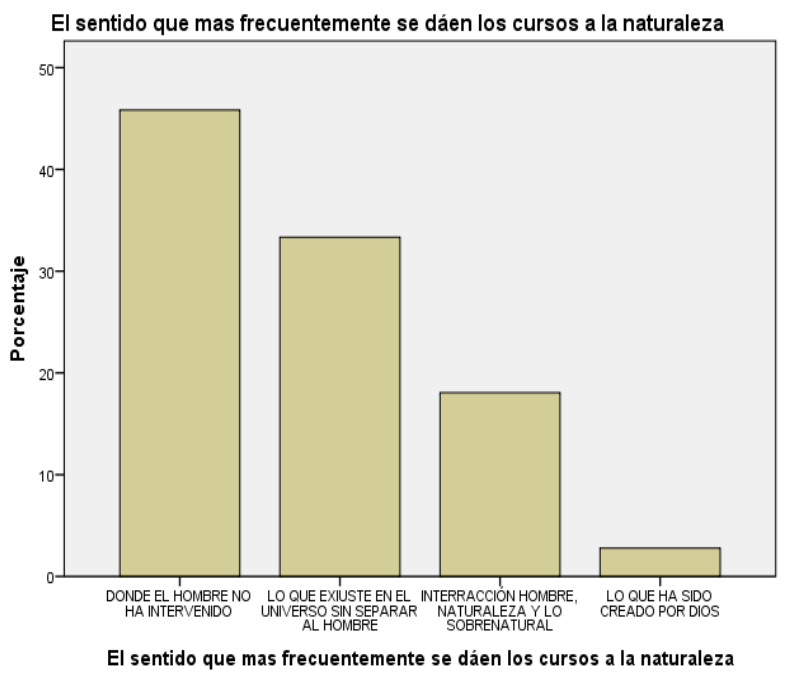

Figura 6. Sentido mas frecuentemente de naturaleza en los cursos.

Según la tabla y Fig. 6 parece que los cursos dan preferencia a dos enunciados: la naturaleza sin incorporar al ser humano y la naturaleza que lo incorpora. Un porcentaje importante llama naturaleza a la interacción del hombre con el entorno físico.

Tabla 7. Qué Entiende por naturaleza - ciclo que estudia.

\begin{tabular}{lccc}
\hline \multirow{2}{*}{ Qué entiende por naturaleza } & \multicolumn{3}{c}{ Ciclo que estudia } \\
& Del & Del & Del \\
& 1 al 3 & 4 al 6 & 7 al 10 \\
\hline Lo no creado por el hombre & 5 & 85 & 79 \\
Nosotros y todo lo que nos rodea & 3 & 14 & 11 \\
Lo natural & 0 & 1 & 0 \\
Algo propio & 1 & 0 & 0 \\
Espacio donde nos encontramos & 1 & 5 & 3 \\
Interacción hombre-naturaleza & 0 & 4 & 0 \\
Sistema vivo & 0 & 4 & 0 \\
Esencia donde se desarrolla el & 0 & 0 & 2 \\
hombre & 0 & 2 & 0 \\
Creación de Dios & 0 & 1 & 1 \\
No responde & & & \\
\hline
\end{tabular}




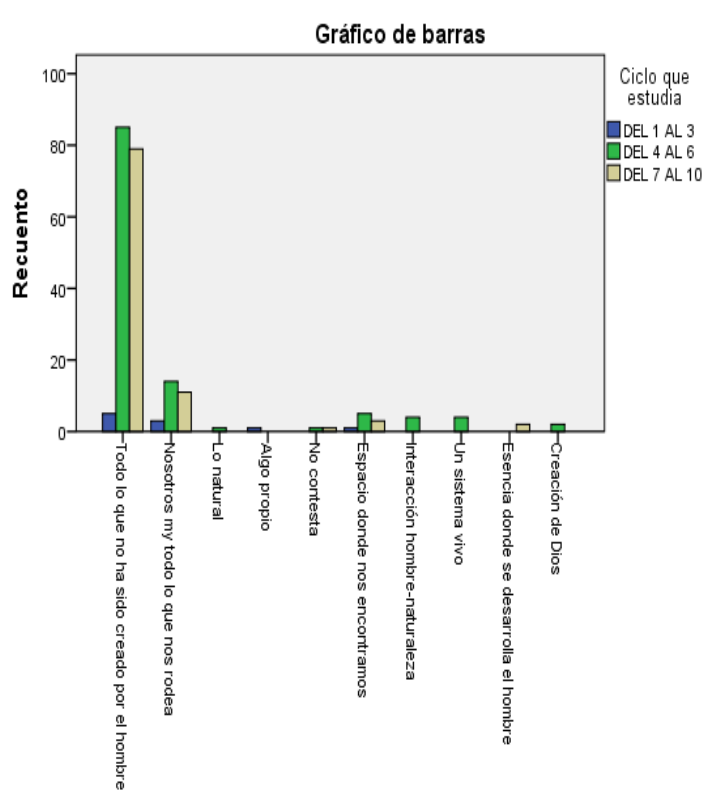

Figura 7. Qué entiende por naturaleza - ciclo que estudia.

El cruce de variables: qué entiende por naturaleza-ciclo que estudia, (tabla 7) presenta la misma composición que las respuestas anteriores, en las tres agrupaciones de ciclo lo no creado por el hombre obtiene mayores respuestas seguido por la que incorpora al hombre. Comparando el total de respuestas por grupos el intermedio es el de mayor heterogeneidad.

El impacto de los estudios universitarios es muy pobre para la mayoría de los estudiantes respecto a cambios en su concepción de naturaleza. La tabla 8 muestra que para el $64.1 \%$ no hubo cambios. Solamente una minoría $(35.9 \%)$ acepta que gracias a sus estudios pudo modificar su concepción. En el focus group donde también se hizo esa averiguación se propuso como posible respuesta una abierta resistencia a recepcionar otras ideas por motivos culturales, sin embargo se encontró una respuesta más endogámica: aceptación sin mayor discusión en la mayoría de cursos, de lo que señalan los textos.

Tabla 8. La universidad hizo cambiar su concepción de naturaleza.

\begin{tabular}{ccc}
\hline & Frecuencia & \% Válido \\
\hline $\mathrm{Si}$ & 79 & 35,9 \\
$\mathrm{No}$ & 141 & 64,1 \\
\hline
\end{tabular}

Tabla 10. Es pertinente que en la UNALM se precise un concepto de naturaleza.

\begin{tabular}{ccc}
\hline & Frecuencia & \% Válido \\
\hline SI & 132 & 59,7 \\
NO & 89 & 40,3 \\
\hline
\end{tabular}

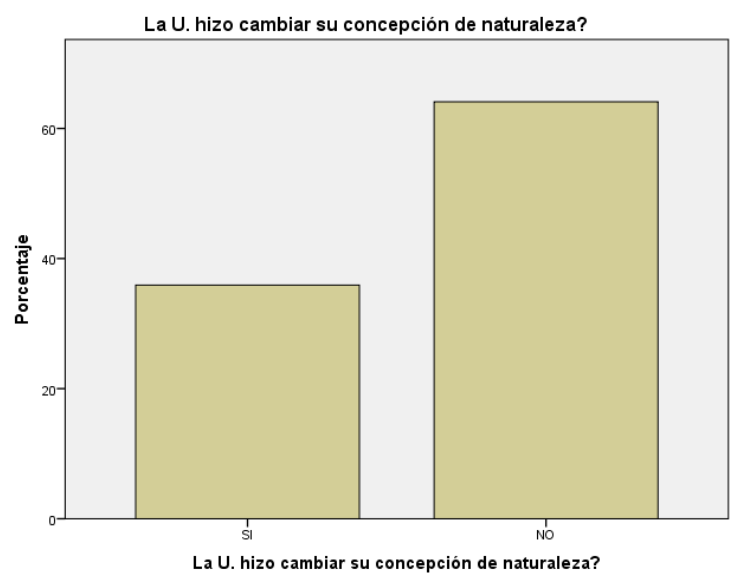

Figura 8. La universidad hizo cambiar su concepción de naturaleza.

Más del $50 \%$ de estudiantes que respondieron haber modificado su concepción de naturaleza en la universidad, (tabla 9), plantean dos grandes grupos de respuestas:

a). Uno que se puede denominarla conservacionista: el cambio les permite cuidar mejor la naturaleza (33.3\%).

b). Otro grupo está referido a la aceptación de otras visiones: se sienten ahora parte de la naturaleza (20.6\%). Si se adiciona la respuesta "naturaleza más que árboles, selva o animales", (12.7\%), asumiendo que también incorpora al hombre, llega a tener el mismo porcentaje (33.3\%) que el primer grupo.

El $59.7 \%$ de encuestados cree que es pertinente que la universidad precise un solo concepto de naturaleza (tabla $10)$, para la mayoría de ellos $(54.2 \%)$ debe ser concebida como el área, el espacio físico, donde el hombre no ha intervenido como está planteada en la tabla 11. La misma tabla muestra también que un $10.3 \%$ cree que el concepto que caracterizaría a la UNALM es aquel que incorpora al hombre en la naturaleza.

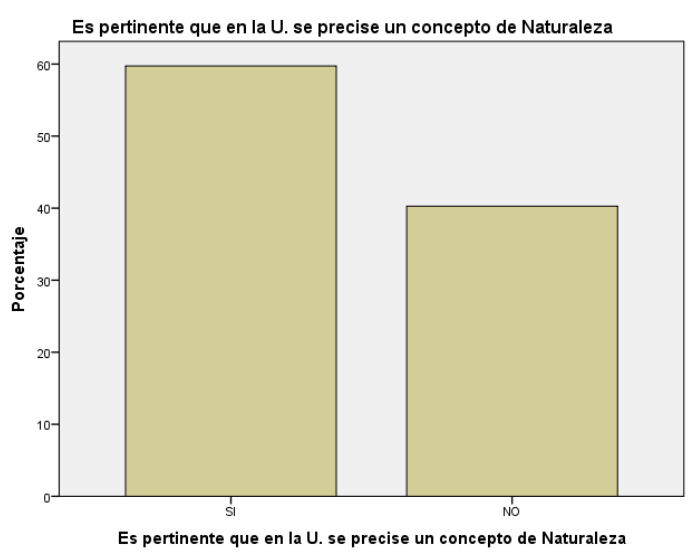

Figura 10. Es pertinente que en la UNALM se precise un concepto de naturaleza. 


\section{Conclusión}

Para los fines académicos la conclusión más importante del estudio señala que entre los estudiantes de la UNALM predomina el concepto de naturaleza adscrito al paradigma occidental: la naturaleza totalmente separado del hombre. Este predominio es notorio tanto entre los encuestados $(76.1 \%)$, como entre los que participaron del focus group. En este último caso cuando se trató sobre qué institución o quiénes tuvieron mayor influencia en la formación de sus opiniones sobre la naturaleza los comentarios mayoritarios fueron: la escuela, el colegio, mi familia, los viajes, entre otros, con ninguna mención a los cursos o a la universidad. La tabla 11, donde se averigua si es pertinente que la universidad maneje un concepto único de naturaleza y cuál sería ese concepto, el 54.2\% de encuestados señalan: "lo que hay sin intervención del hombre", lo que confirma el tremendo peso que tiene esa acepción entre los estudiantes. Una posible explicación a este fenómeno se encuentra al averiguar cuál es el sentido de naturaleza que se da en los cursos (tabla 6), el 45,8\% responde "donde el hombre no ha intervenido".

Sin embargo, el estudio también refleja una posición alternativa a la visión occidental. Para un significativo número de estudiantes la naturaleza está compuesta por todos los elementos del universo incluyendo al hombre, como se puede ver en la tabla 5 , el $12.6 \%$; tabla $6,33.3 \%$. Igualmente en la tabla 7, en los tres grupos de ciclos esta respuesta es la segunda en importancia. Las respuestas que se encuentran en las tablas 9 y 11 van en el mismo sentido, en el primer caso el $20.6 \%$ de encuestados señala que uno de los cambios que encuentra en el concepto es el "incluirme como parte de la naturaleza" y en el segundo caso el $10.3 \%$ señala que el concepto de naturaleza que debe manejar la universidad es el del "universo total incluido el hombre".

Tanto en la encuesta como en el focus group se ha preguntado si están de acuerdo o no con el siguiente párrafo a fin de verificar la coherencia de las respuestas:

"Las culturas que más avanzaron son aquellas que consiguieron dominar a la naturaleza gracias al desarrollo de la ciencia y la tecnología, contrariamente las que avanzaron poco o no avanzaron son las que no llegaron a dominar a la naturaleza debido a que no lograron (o lo lograron a un nivel muy bajo) el desarrollo científico y tecnológico".

Tabla 13 Esta de acuerdo o en desacuerdo con el enunciado

\begin{tabular}{ccc}
\hline & Frecuencia & \% Válido \\
\hline De acuerdo & 117 & 52,9 \\
En desacuerdo & 104 & 47,1 \\
\hline
\end{tabular}

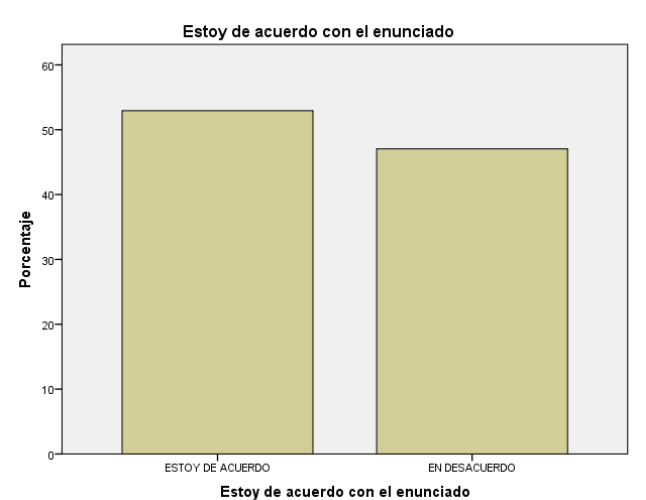

Figura 13. Esta de acuerdo o en desacuerdo con el enunciado.

En ambos casos la mayoría de las respuestas están de acuerdo en que el hombre o las sociedades deben dominar a la naturaleza, lo que confirma la visión dicotómica: el hombre versus la naturaleza. En el caso de las encuestas (tabla 12), el 52.9\% de los encuestados aceptan el enunciado como sucedió con la mayoría de los participantes en el focus group.

Finalmente se han puesto las respuestas tal como han sido dadas por los estudiantes, mostrando la diversidad de contenidos, algunos de ellos contradictorios, sin modificaciones ni amputaciones, evitando caer en una coherencia postiza que pueda disminuir el valor de la investigación.

\section{Literatura citada}

Boff. 1997. Leonardo. Ecología: grito de la Tierra, grito de los pobres. 1997.

Bordehore, C.------Problemas ambientales y problemas humanos. https://www.google.com.pe/?gfe_rd=cr\&ei=F4dU7uBM-_d8gfuwoGoDQ\#q=BORDEHORE $\% 2 \mathrm{C}+\mathrm{C}$ $\% \mathrm{C} 3 \%$ A 9 sar. + Problemas + ambientales $+y+$ problemas $+h$ umanos

Caceres, E. 2005. Arguedas: entre el sentir, pensar y saber. Herencia teórica para entender en Ande. En Arguedas y el Perú de hoy. Carmen María Pinilla, Editora General. 2005. SUR Casa de Estudios del Socialismo.

Cerpa, B. 1966. Aspectos teóricos e históricos de la relación sociedad y naturaleza. En Tierra Nuestra. Año 6, No. 3 y 4, Lima, 1966. Revista del Departamento De ciencias Humanas, UNALM.

Descola, P. 1998. Las cosmologías de los indios de la amazonía. 1998

Descola, P.2001. Construyendo Naturalezas. Ecología simbólica y práctica social. En Naturaleza y Sociedad. Perspectivas antropológicas. Coordinado por Philippe Descola y Gísli Pálsson. Ed. S. XXI. 2001. 101-123.

Descola, P. y Gísli, P.2001. Introducción. En Naturaleza y Sociedad. Perspectivas antropológicas. Coordinado por Philippe Descola y Gísli Pálsson. Ed. S. XXI. 2001. 11- 
33.

Chirif, A.----. Presente y futuro de los pueblos amazónicos.

http://ibcperu.org/doc/isis/8893.pdf s/f

Gasché, J. y Vela, N. 2012. Sociedad Bosquesina. Tomo I. 2012

Gudynas, E. 2004. Ecología, Economía y Ética del Desarrollo Sostenible. 2004

Heyd, T. 2011. Pensar la relación entre cultura y cambio climático. En Perspectivas Culturales del clima. Astrid Ulloa, Editora. ILSA, Universidad nacional de Colombia. 2011.17-30.

Kwiatkowska, T.2006. Lo natural: Un concepto enigmático. Ludus Vitalis, vol. XIV, num. 25, 2006. 153161.

Lopez, S.2005. Modos de racionalidad en Todas las Sangres. En Arguedas y el Perú de hoy. Carmen María Pinilla, Editora General. 2005. SUR Casa de Estudios del Socialismo.

Moran, E. F. 1990. A Ecología humana das populacoes da amazonía. Petrópolis; RJ: Vozes, 1990.

Pálsson, G. 2001. Relaciones Humano-Ambientales. Orientalismo, paternalismo y comunalismo. En Naturaleza y Sociedad. Perspectivas antropológicas. Cordinado por Philippe Descola y Gísli Pálsson. Ed. S. XXI. 2001. 80-100.

Reboratti, C. 2000. Ambiente y sociedad. Conceptos y relaciones. Ariel. Buenos Aires. 2000.

Rivera, J. 1996. Pensamiento Amazónico: sobre naturaleza, sociedad y hombre. 1996

Trazegnies, F. 1997. Liberalismo y posmodernidad. En: El Perú en los albores del siglo XXI. 1997. 91-123.

Sempere, J. y Riechmann, J. 2000. Sociología y medio ambiente. 2000

Schumacher, E. F. 1986. Lo Pequeño es Hermoso. Ediciones Orbis, S. A. 1986

Tiwi Paati, F.--- El territorio y los derechos de los pueblos indígenas. s/f

Varas, N. y Williams, M. 2010. Los rituales para superar conflictos en los ecosistemas campesinos. En Revista Tierra Nuestra, Departamento de ciencias Humanas, UNAL. 2010.

Vilcapoma, J. C. 2008. La danza a través del tiempo en el mundo de los Andes. 2008 